\title{
BLIND SOURCE SEPARATION TECHNIQUE FOR LINEAR MIXTURES BASED ON ACCURATE ESTIMATION OF PROBABILITY DENSITY FUNCTION
}

\author{
M. F. FAHMY, U. S. MOHAMMED AND N. A. BAHRAN \\ Department of Electrical \& Electronics Eng., Faculty of Eng., Assiut \\ University, Egypt \\ Phone: + 20-10-1346626, Fax: + 20-88-2332553 \\ Fahmy@aun.edu.eg,Usama@aun.edu.eg
}

(Received July 20, 2010 Accepted September 20, 2010)

\begin{abstract}
This paper presents an accurate nonparametric method for evaluating signal's probability density function ( $p d f)$, as well as its entropy. It is based on using Bspline wavelets, as the smoothing filter for the data histogram distribution. Due to the excellent energy concentration feature of Bspline wavelets, this estimation was found to be accurate and robust of probability density function ( $p d f)$ estimation that are needed in some linear blind source separation (BSS) designs. The validity of the proposed technique is checked by its ability of recovering linearly blind source BSS, with a simple check to verify exact source recovery when no information is available about the mixing system. Several experiments have been carried out, to verify the ability of the proposed technique to accurately estimating signal's pdf as well as recovering linearly mixed signals and images with a simple independency check to determine whether exact separation is achieved or not.
\end{abstract}

\section{INTRODUCTION}

The blind signal separation (BSS) is a well-established statistical signal processing technique that aims at decomposing a set of multivariate signals or images, into a set of statistically independent/components minimal loss of information content. The BSS problem arises in many fields of studies, including speech processing, data communication, biomedical signal, etc, where both the sources as well as the transmitting media characteristics, are unknown. Blind source separation amounts to recovering unknown source si(n),i=1,2.., $\mathrm{m} n=1,2, . . \mathrm{N}$ from a number of observations $x i(n), i=1,2, . . p$, which are unknown functions of the sources. The sources are assumed to be mutually independent and no information exits about their distribution. This subject has been extensively studied for the linear memoryless mixture, where separation algorithms are mainly based on Independent Component Analysis (ICA), $[1-7,11]$. The basic concept of ICA techniques stems from the fact that separation is achieved if the output's negentropy is maximized. Negentropy for a signal $y(n)$ is defined as $\mathbf{J}$, where $\mathbf{J}=\operatorname{Ent}($ ygaus)-Ent(y), Ent(.) denotes entropy, ygaus is a Gaussin random variable having the same length and variance as $\mathrm{y}$. Negentropy is used because of its scale invariance property. It is approximated using either the kurtosis and higher order statistics functions or nonlinear approximation of the entropy functionss, [7]. 
The kurtosis and higher order statistic techniques suffer from nonrobustness and noise sensitivity, whereas expanding negentropy by nonlinear function is not unique.

In this paper, an accurate nonparametric technique is proposed for pdf, score function evaluations [8-9], as well as entropy estimation. It is based on smoothing histogram distribution by Bspline wavelet. Bspline wavelets are used due to its superior de-noising properties, [10,12]. Having accurately estimated the signal's pdf, we are now able to accurately estimate the output's negentropy, and subsequently perform BSS. Besides having a simple check whether independent components have been obtained or not, several simulation examples have been given to show that the proposed technique manages to accurately estimate of pdf, score and entropy functions. Further, it is used in BSS of linearly mixed sound and images. Separation quality is checked by showing that the convolution of the pdf of any output pair, almost coincides with the distribution of their sum. Comparisons with classical nonlinear ICA techniques, have also verified the superior performance of the proposed pdf estimation technique.

\section{BSPLINE BASED WAVELET FAMILY}

In [13-14], a B-Spline based wavelet family has been constructed. It has been shown that for an $m^{\text {th }}$ order Bspline polynomial $b_{m}(t)$, the scaling analysis filter $P(z)$, is given by

$$
P(z)=\left[\frac{1+z^{-1}}{2}\right]^{m} \quad z=e^{j \frac{\omega}{2}}
$$

Whereas the high-pass filter, $Q(z)$ is given by

$$
\begin{aligned}
& Q(z)=z^{-(2 m-1)} P\left(-z^{-1}\right) A(-z) \\
& A(z)=\sum_{k=-m+1}^{2 m-1} b_{2 m}(k) z^{-k} \\
& =(-z)^{-(m-1)} \tilde{P}(-z) A(-z) \\
& \widetilde{P}(z)=z^{-m} P\left(z^{-1}\right)=-E(-z) \tilde{P}(-z) \\
& E(z)=z^{-(m-1)} A(z)
\end{aligned}
$$

This concludes the decomposition step. Note that both of $P(z)$ and $Q(z)$ are FIR filter of lengths $m+1$ and $2 m$, respectively. As far as the synthesis filter banks $G_{0}(z)^{\prime}$ $G_{l}(z)$ are given by

$$
G_{O}(z)=z \frac{E(z)}{E\left(z^{2}\right)} \widetilde{P}(z), G_{1}(z)=z \frac{P(z)}{E\left(z^{2}\right)}
$$

This concludes the synthesis step. In [14], the pairs $\left\{P(z), Q(z), G_{O}\left(z^{-1}\right)\right.$ and $\left.G_{1}\left(z^{-1}\right)\right\}$ constitute a perfect reconstruction (PR) system.

Moreover, it also shown how to approximate the IIR non-causal symmetric functions $G_{0}(z) \& G_{I}(z)$, by an equivalent FIR filters $F_{0}(z) \& F_{I}(z)$, up to any prescribed accuracy. Figure 1 shows the complete 1-level PR Bspline wavelet system. 


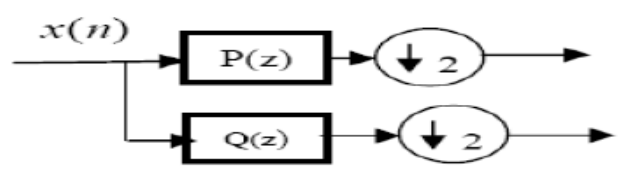

(a)

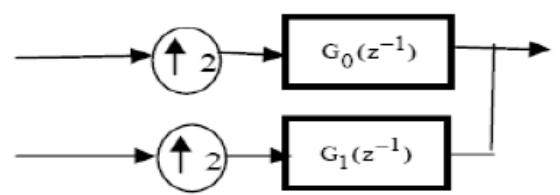

(b)

Fig.1. (a) Bspline based analysis bank (b) Synthesis bank

\section{DENISTY \& SCORE FUNCTIONS ESTIMATION USING B- SPLINE WAVELETS}

Due to the distinctive feature of the Bspline wavelets that have a large amount of energy concentration with low spatial frequencies, it is efficient in signal and image denoising. As a specific important application, we try to estimate the probability density function $p d f$ of a signal $x(t)$. The classical histogram method can not be relied upon in methods requiring precise estimation of the $p d f$ and signal entropies. The proposed Bspline based $p d f$ estimation is described as follows:

1. Obtain a rough estimation of the $p d f$ using $N_{B}$ bins, for example by using the Matlab hist function. Denote it by $h(k), k=1,2, \ldots, N_{B}$

2. Decompose $h(k)$ using $n$-level Bspline wavelet. Set the detail coefficients to zero

3. Reconstruct $h(k)$, using the Bspline reconstruction wavelets.

As a simulation example, we estimate the $p d f s$ of unity variance Uniform, Rayleigh, Gaussian and Exponential random variable distributions. The test signal is 4096 samples long. Cubic Bspline wavelet with $N_{B}=128$ and 3-levels Bspline wavelets is used. The test examples were run for 10 different seeds. Table 1, compares the \% error norms between the theoretical and the estimated $p d f$, using cubic Bspline $e_{b s p}$ and $7 / 9$ Biorthogonal wavelets $e_{7 / 9}$ for different decomposition levels $n$. The table gives also the theoretical and estimated entropy $E_{n t p} .(=-p \log p)$, for these cases. These results generally indicate that, except for the uniformly distributed random variable, the best decomposition level occurs at minimum entropy. Fig. 2-a shows the theoretical and estimated $p d f^{\prime} s$ for the best decomposition level of these 4 cases. Moreover, in order to verify the robustness of the proposed Bspline technique for $p d f$ estimation even in the presence of noise, 20000 samples of the speech signal sampled at $12 \mathrm{KHz}$ is used. The speech is contaminated with AWGN to yield $S N R=10 \mathrm{~dB}$. Fig.2-b, shows the effect of noise on the $p d f$ estimation for both Bspline and 7/9 Biorthogonal 4-level of the wavelet and with $N_{B}=128$.

Now, having accurately estimated the $p d f$ distribution $p_{x}(x)$, the score function $\psi_{x}(x)$ defined as

$\psi_{\mathrm{x}}(\mathrm{x})=\frac{\frac{\partial \mathrm{p}_{\mathrm{x}}(\mathrm{x})}{\partial \mathrm{x}}}{\mathrm{p}_{\mathrm{X}}(\mathrm{x})}=\frac{\partial \log \mathrm{p}_{\mathrm{x}}(\mathrm{x})}{\partial \mathrm{x}}$

It is evaluated as follows:

1. For the batch $\mathrm{x}(\mathrm{n})$, evaluate $\mathrm{px}(\mathrm{x})$, as described above using NB histogram bins 
2. Evaluate $y(n)=\log \left(p_{x}(x)\right), n=1,2, \ldots N_{B}$

3. The score function is approximated as $\psi_{x}\left(x_{n}\right)=(y(n)-y(n-1)) / \Delta \Delta x, n=1,2, \ldots, N_{B}, y(0)=0$

$\Delta x$ is the spacing between the histogram bins

Table 1 Comparison for different $\mathbf{n}$ levels decomposition, the relative \% error norms ebsp \& e7/9 between the theoretical and estimated pdf when using cubic BSpline and 7/9 Biorthogonal wavelet.

\begin{tabular}{|c|c|c|c|c|c|c|c|c|c|c|c|c|}
\hline \multirow[t]{2}{*}{$n$} & \multicolumn{3}{|c|}{$\begin{array}{c}\text { Uniform } \\
\text { Theor. Entropy }=\ln 2\end{array}$} & \multicolumn{3}{|c|}{$\begin{array}{c}\text { Rayleigh } \\
\text { Theor.Entropy } \\
=1.0587\end{array}$} & \multicolumn{3}{|c|}{$\begin{array}{c}\text { Gaussian } \\
\text { Theor.Entropy } \\
=1.4189\end{array}$} & \multirow{2}{*}{\multicolumn{3}{|c|}{$\begin{array}{c}\text { Exponential } \\
\text { Theor.Entropy }=1 . \\
E_{\text {trp }} \% e_{b s p} \% e_{7 / 9}\end{array}$}} \\
\hline & & & & $E_{t r p} \%$ & $e_{b s p}$ & $\% e_{7 / 9}$ & $E_{t r p}$ & $\% e_{b s p}$ & $\% e_{7 / 9}$ & & & \\
\hline 1 & 0.6784 & 0.1180 & 0.1687 & 0.9428 & 0.0935 & 0.1324 & 1.4233 & 0.0802 & 0.1163 & 1.0230 & 0.0647 & 0.0867 \\
\hline 2 & 0.6816 & 0.0880 & 0.1555 & 0.9332 & 0.0704 & 0.1171 & 1.4199 & 0.0631 & 0.1103 & 1.0058 & 0.0396 & 0.0709 \\
\hline 3 & 0.6833 & 0.0653 & 0.1561 & 0.9428 & 0.0521 & 0.1066 & 1.4214 & 0.0421 & 0.1011 & 1.0090 & 0.0437 & 0.0795 \\
\hline 4 & 0.6813 & 0.0886 & 0.1460 & 0.9430 & 0.0424 & 0.1160 & 1.4202 & 0.0241 & 0.1058 & 1.0105 & 0.0659 & 0.1134 \\
\hline 5 & 0.6765 & 0.1293 & 0.1541 & 0.9705 & 0.1117 & 0.1851 & 1.5311 & 0.1245 & 0.1986 & 1.3851 & 0.2932 & 0.2710 \\
\hline 6 & 0.6752 & 0.1384 & 0.1549 & 1.2069 & 0.3169 & 0.2897 & 1.9265 & 0.5619 & 0.5904 & 1.6527 & 0.5165 & 0.2807 \\
\hline 7 & 0.6792 & 0.1116 & 0.1612 & 1.4196 & 0.5917 & 0.6405 & 2.0021 & 0.6845 & 0.7178 & 1.9220 & 0.6985 & 0.6391 \\
\hline
\end{tabular}
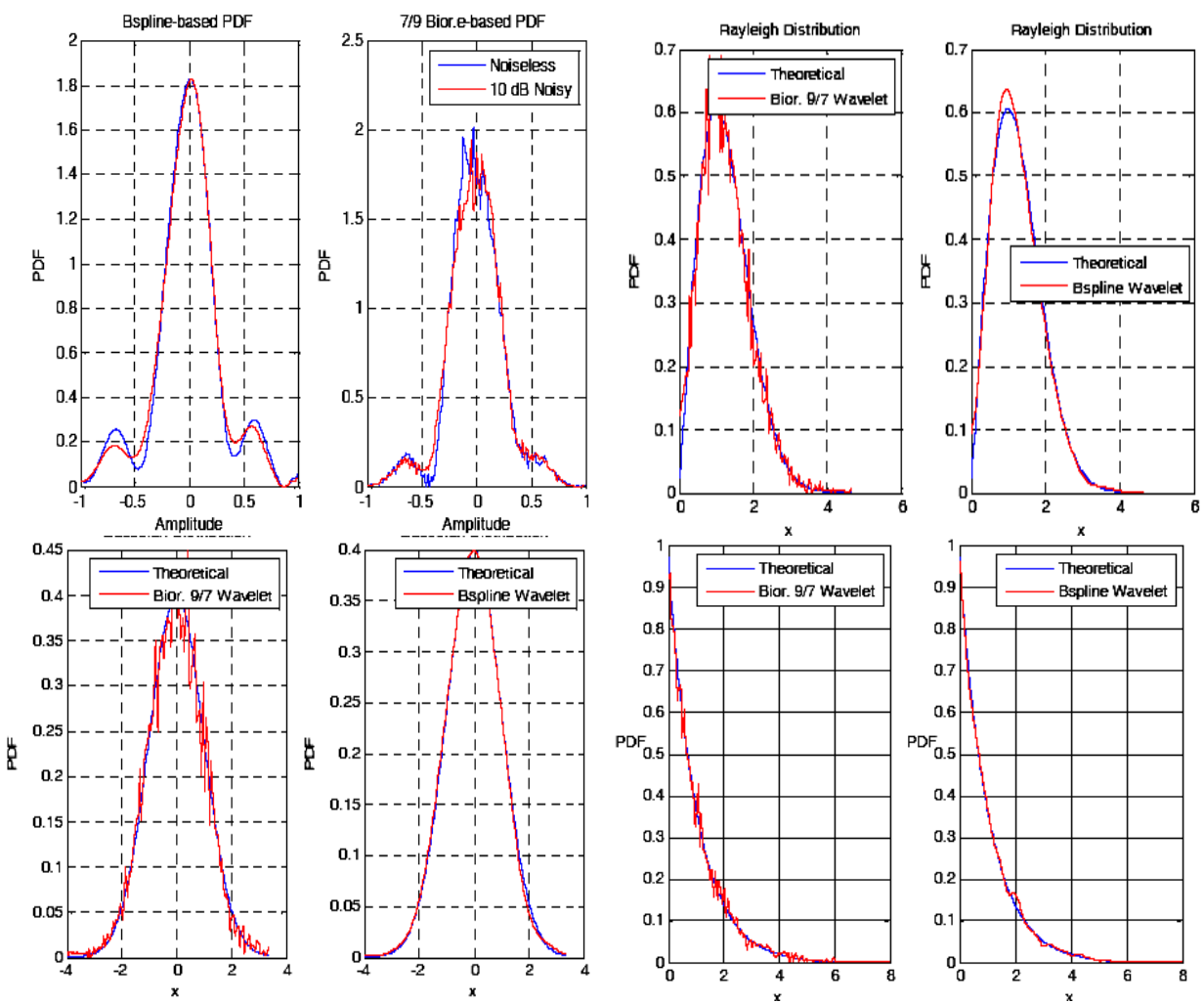

(a) 

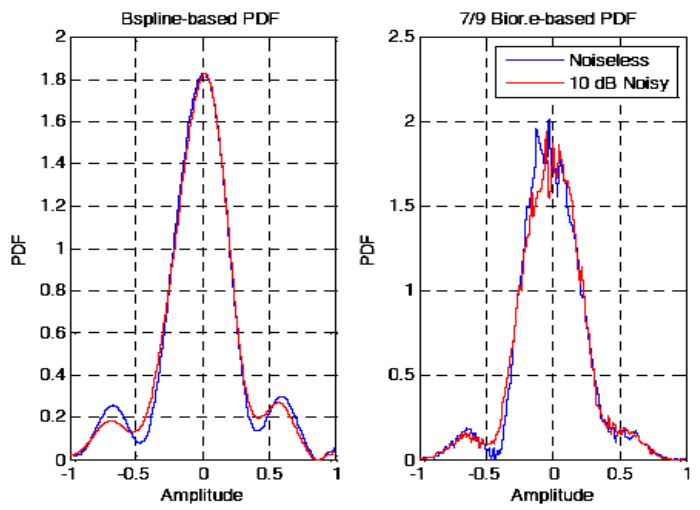

(b)

Fig. 2.(a) Comparisons of theoretical Bspline and Biorthogonal wavelet-based $p d f$ estimation of Uniform, Rayleigh, Gaussian, and Exponential distributions. (b), shows the effect of noise on PDF estimation for both Bspline and 7/9 Biorthogonal 4-level wavelets. The noisy signal is $10 \mathrm{~dB}$ speech signals.

As an illustrative example, we consider the example cited in [9]. A batch of 2048 zero-mean, unity variance uniformly distributed in the interval $[\sqrt{3} \sqrt{3}]$. The proposed Bspline based score function is evaluated using 3-levels Bspline wavelets with $N_{B}=128$. Fig. 3-a, compares the theoretical score function with Gram-Charlier approximation, MSE approximation method of [9], and the proposed Bspline estimation. Fig.(3-b), does the same comparison for 2048 exponential distribution unity variance random variables. In both cases, the improvement achieved by using the proposed Bspline-based technique over other approaches, is clear.
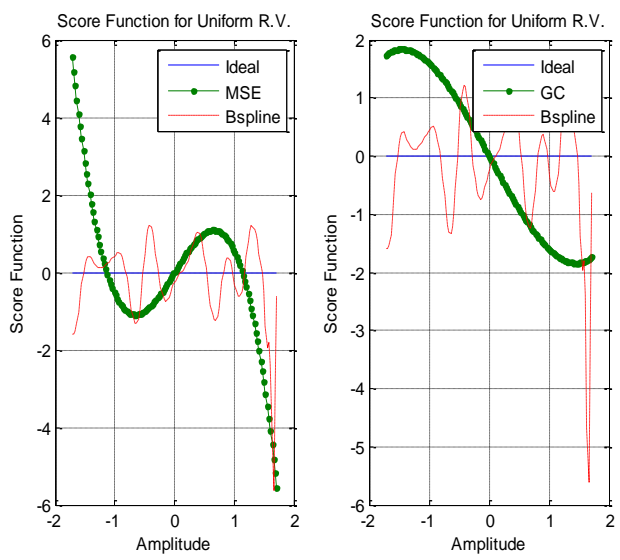

(a)
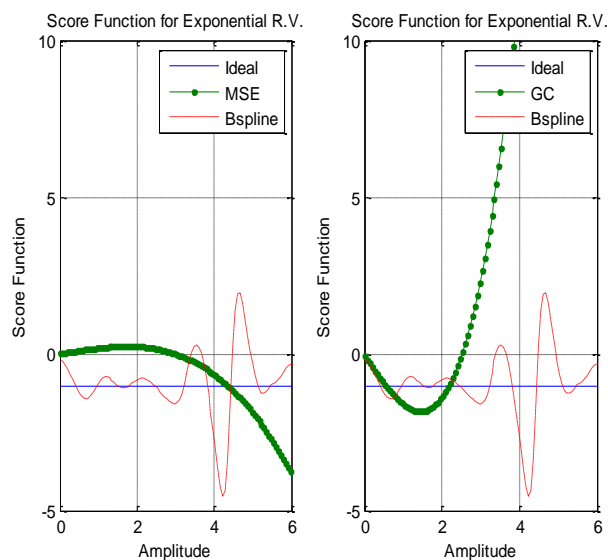

(b)

Fig.3. Score function of uniformly distributed random variables in (a) and exponentially distributed random variables in (b) 


\section{APPLICATIONS IN BSS OF LINEAR MIXTURE}

The validity of the proposed Bspline density estimation is checked by using it in estimating independent components in linear BSS mixture. Besides, an important asset of this technique lies in its ability to provide a simple check for ensuring signal separation without any prior knowledge about the mixing system. Two approaches can be used to achieve this goal. They are the $p d f$ approach and the negentropy technique. The $p d f$ technique, is based on the fact that if $y_{1}, y_{2, \ldots}, y_{m}$ constitute an independent set, then

$$
p_{w}=p\left(y_{i}\right) \quad p\left(y_{j}\right), w=y_{i}+y_{j i \neq j,(i, j) \in(1, m)}
$$

Thus, if the mixed data vector $\boldsymbol{x}$ is distributed according to the ICA model

$\boldsymbol{x}=\boldsymbol{A} s$, where $s=\left[\begin{array}{llll}s_{I}(t) & s_{2}(t) & \ldots & s_{m}(t)\end{array}\right]^{T}$ represents the independent sources and $\boldsymbol{A}$ is a constant nonsingular $m x m$ mixing matrix. So, to recover $s$ from the mixed signals $\boldsymbol{x}$, a decoupling system $B$ should be designed to make the output components $y$, $y=B x$, constitute an independent set. Independency is ensured by finding $B$ that minimizes the distance between both sides of the above equation, for all possible combinations of the outputs.

As far as the second entropy technique, it is well known that due to the central limit theory, the distribution of the sum of linearly mixed independent random variables tends toward Gaussian distribution. Thus, the decoupling system should ensure that the distribution of $y$ is as far as possible from Gaussiadistribution. This feature is known as nongaussianity, [7]. Nongaussianity is measured in terms of negentropy.

The suggested proposed separation algorithm combines both techniques. The optimum decoupling matrix B, should minimize the following objective function

$$
\zeta=\sum_{k=1}^{m} e^{-J_{k}}+\sum_{i \neq j}^{m} p_{y_{i j}} a b s\left(\log \left(\frac{p_{w}}{p_{y_{i j}}}\right)\right) \quad w=y_{i}+y_{j}, i, j=1,2, . ., m
$$

Now, since any nonsingular matrix $B$ is decomposed as $B=Q R, Q$ is an orthogonal matrix and $R$ is an upper triangular matrix. The determination of $Q$ proceeds as follows:

1. Whiten thr received mixed data $\boldsymbol{x}$ to give $z w$

2. Find the optimum $m$ rotation angles of Given's rotation mxm orthogonal section that maximizes the output negentropies of Eq.(7), when evaluated using the proposed Bspline pdf and entropy estimation.

3. Check independency by checking the distance between both sides of Eq.(6), i.e. the Kullibeck-Leiber (KL) divergence meaure of divergence of [7]. If the error is not acceptable, find the optimum $(m(m-1) / 2)$ parameters of an upper triangular matrix $R$, that maximizes the negentropy or the KL divergence measure.

As an illustration example, we consider three 20000 samples speech signals, sampled at $12 \mathrm{KHz}$. The speech signals are mixed using the mixing matrix 
$A=\left[\begin{array}{ccc}1.5 & 0.8 & 0.5 \\ -0.7 & 1.2 & 0.7 \\ 0.6 & 0.8 & 1.35\end{array}\right]$. Negentropies are estimated using the proposed Bspline $p d f$

estimation technique with $N B=128$, and 3-level Bspline wavelets. Fig. (4), shows the original, mixed and recovered speech, as well as signals, as well as the sum and convolution $p d f^{\prime} s \quad p(w), p_{y_{i j}}, w=y_{i}+y_{j} i \neq j$. The figure shows that, except for a possible permutation the proposed $p d f$ and entropy estimation manages to make the output signals almost independent. The following table, compares the entrpies of the whitened mixed signals with those recovered using the proposed neentropy estimation, and those using the nonlinear tanh- function, [7].

\begin{tabular}{|l|l|l|l|}
\hline & Signal \# 1 & Signal \# 2 & Signal \# 3 \\
\hline Whittened mixed Entropy & 1.3963 & 1.4146 & 1.4121 \\
\hline Recovered $p d f$ Entropy Estim. & 1.2624 & 1.3176 & 1.3767 \\
NonliNnear Entropy Estim. [7] & 1.3758 & 1.2640 & 1.3162 \\
\hline
\end{tabular}
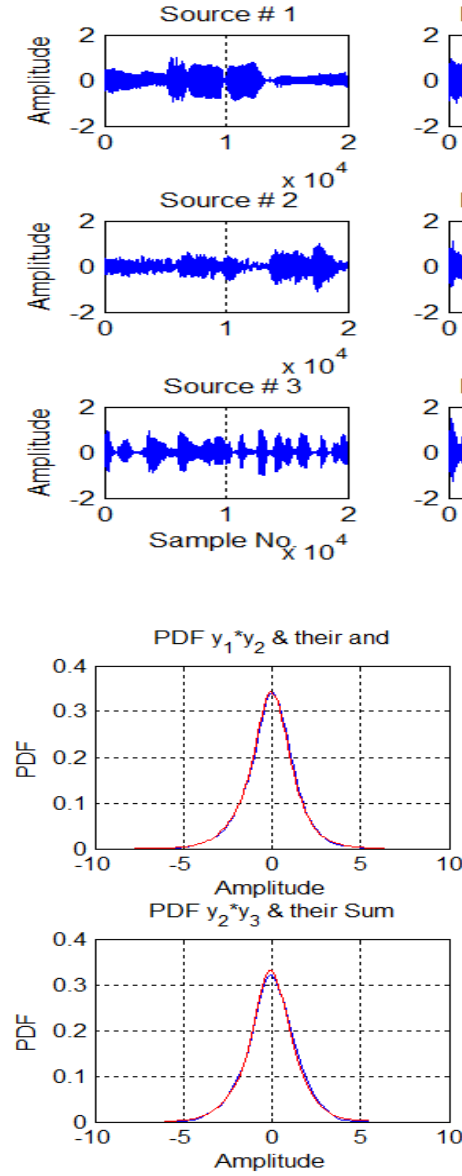
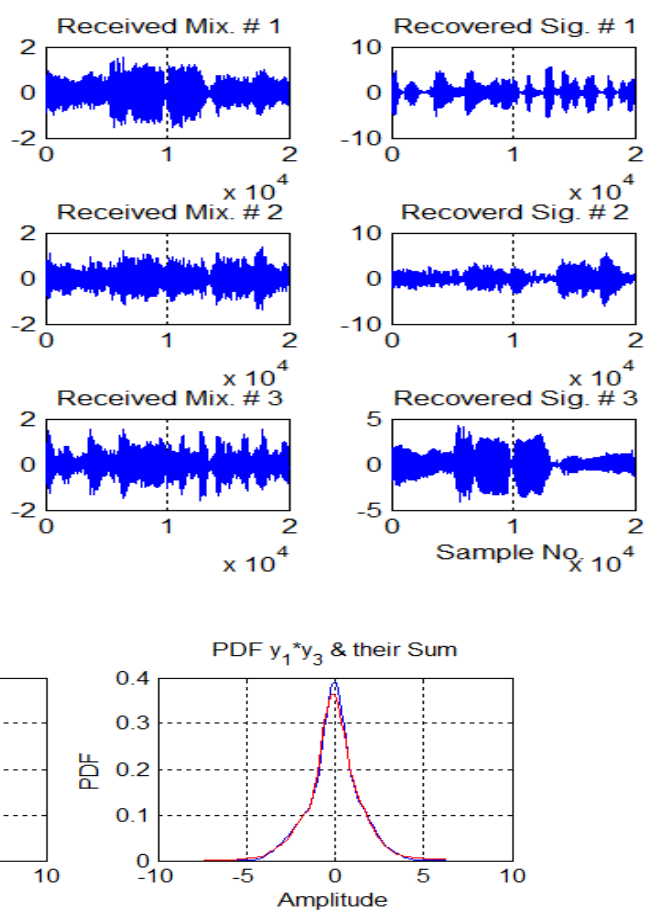

Amplitude

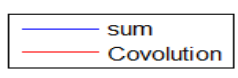

Fig.4: Original, mixed and recovered 3 sound signals, together with the output $p d f$, ie.

$$
p_{z}, p\left(y_{i}\right) \otimes p\left(y_{j}\right), z=y_{i}+y_{j} i \neq j,(i, j) \in(1, m)
$$


Investigation of the resulting distributions, indicate that there is no need for extra upper triangular element minimization. At this point, it is worth mentioning that if the $A$-matrix is convolutive, the negentropy method fails to separate linearly mixed signals, as the convolution operation each source tends to make its density distribution, Gaussian as is demonstrated in fig.5, where the convolutive filter is $4^{\text {th }}$ order FIR.

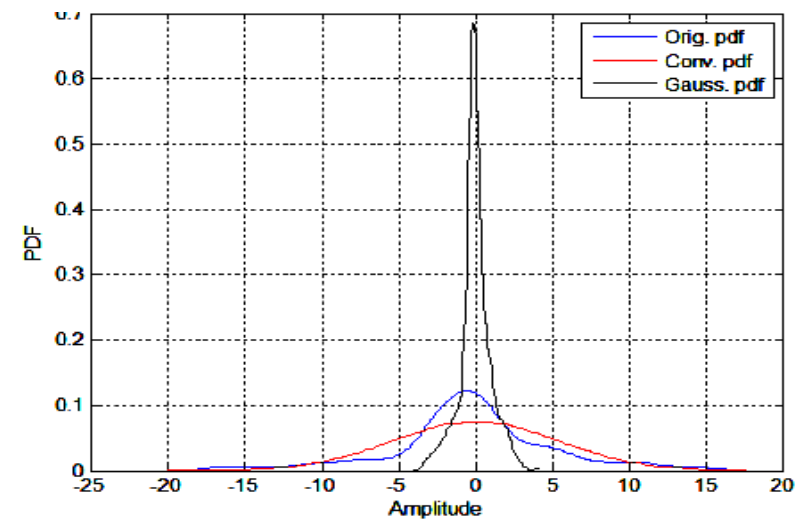

Fig.5. $p d f$ of the original, the convolutive and the Gaussian signal.

As a further check of the proposed $p d f$-entropy estimation, consider its use in separating linearly mixed images. In this respect, two Matlab 256x256 images were mixed using the mixing matrix $A=\left[\begin{array}{ll}0.7 & 0.6 \\ 0.8 & 0.7\end{array}\right]$. The mixed images $X_{i}^{\prime} s, i=1,2$ were converted to vectors. These vectors were whitened. A simple optimization algorithm is constructed to obtain the optimum $Q \& R$ matrices that maximize Eq. (7). The resulting vectors are dc shifted to avoid negative values, and then reshaped to yield 256x 256 images. The proposed entropy estimation was applied using 128 histogram bins together with 3 level Bspline wavelets. Fig. 6 shows the mixed and recovered images of different Matlab images, together with their $p d f$ distribution. The experiment is also repeated using these 3 images that are linearly mixed using the mixing matrix of the sound example. Fig. 7 shows the mixed images as well as the recovered images using the proposed entropy method and the nonlinear ICA method of [7], when using tanh function for estimating negentriopies. The figure shows also the density distribution of the Bspline recovered images, using 128 histogram bins. These results indicate that apart from providing an independency measure, the improvement achieved by the proposed Bspline technique over the nonlinear ICA approach of [7], is clear.

\section{CONCLUSIONS}

This paper, presents an accurate and robust technique for $p d f$ and entropy estimation. As a result, an alternative technique for linear BSS with a simple independency check is proposed. It is shown that this technique works well when the classical kurtosisbased negentropy estimations, or even methods based on their nonlinear expansions, fail to produce acceptable results. The algorithm is tested using different examples (sound signals and natural images) to evaluate its performance. Our extensive 
experiments have confirmed that the use of the proposed procedure provides promising results.
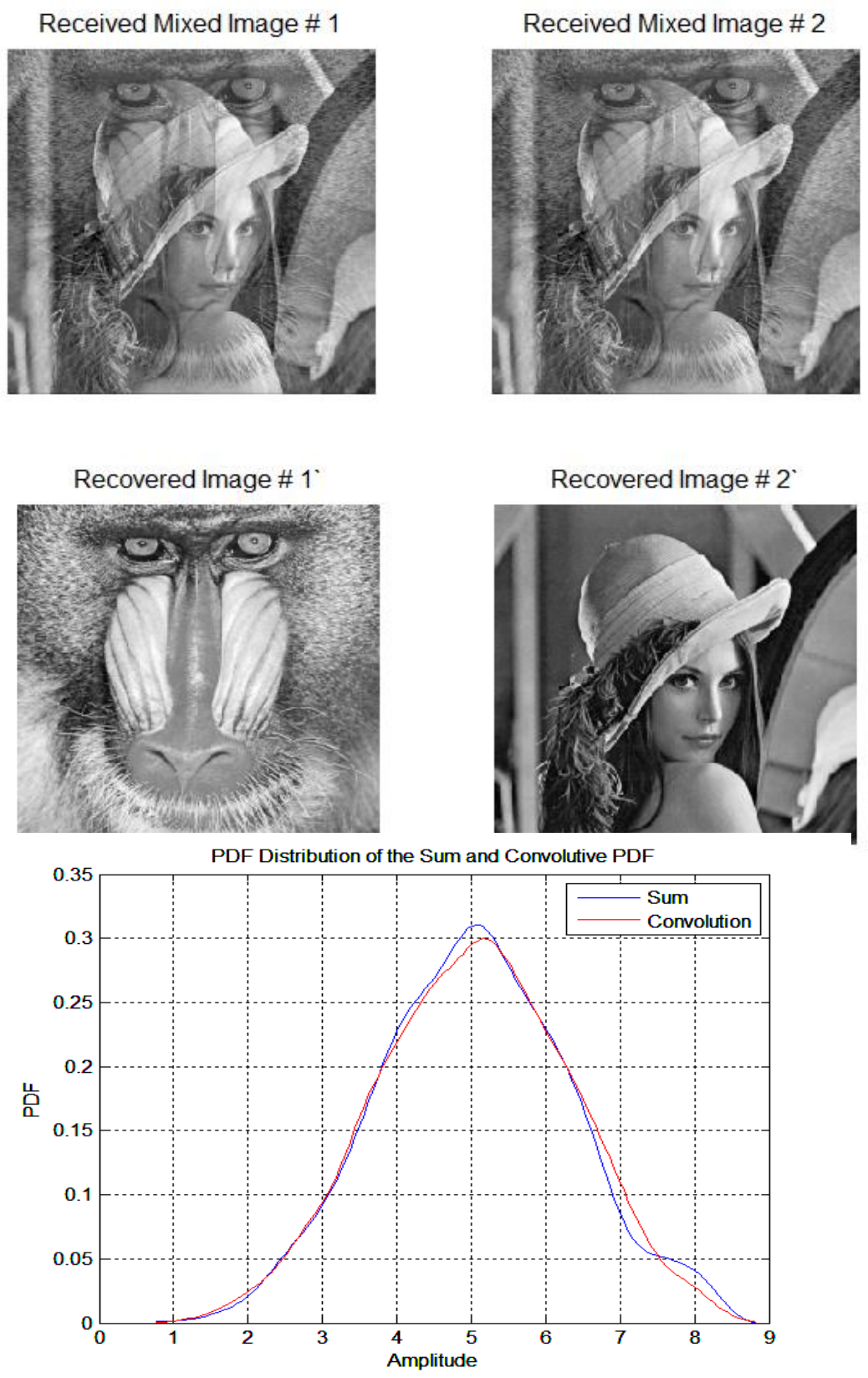

Fig.6 the mixed \& recovered of 2 images and their $p d f$ distribution 


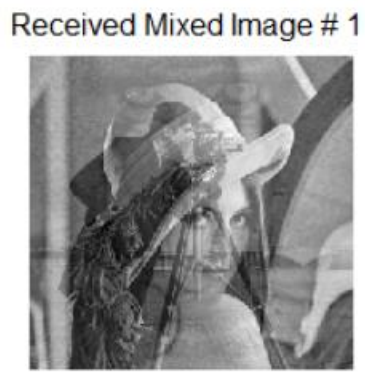

NonLin ICA \#1

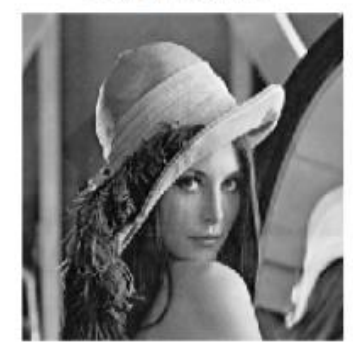

Recovered Image \# 1

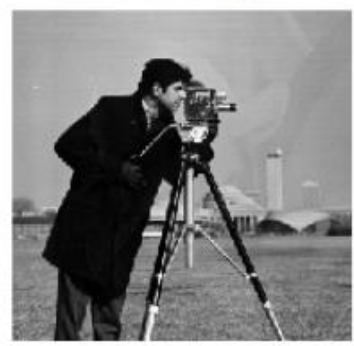

PDF distribution of y1\&y2
Received Mixed Image \# 2

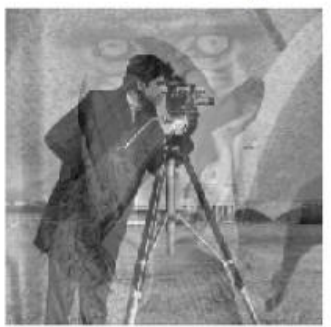

(a)

NonLin ICA \#2

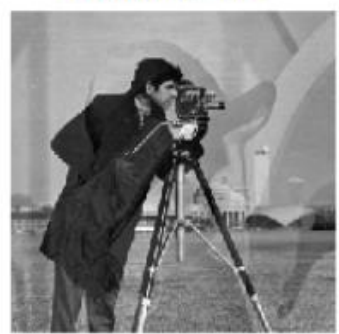

(b)
Received Mixed Image \# 3

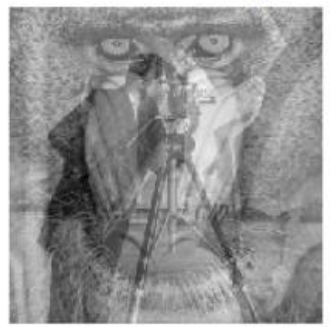

NonLin ICA \#3

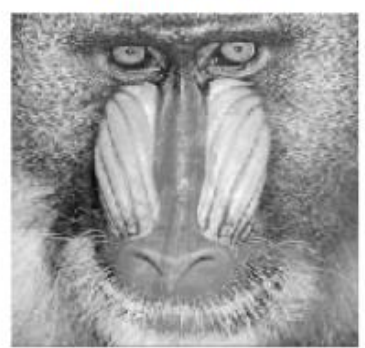

Recovered Image Image \#2

Recovered Image Image \# 3

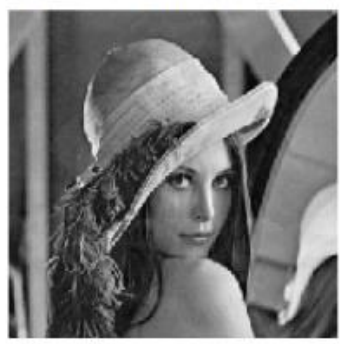

(c)

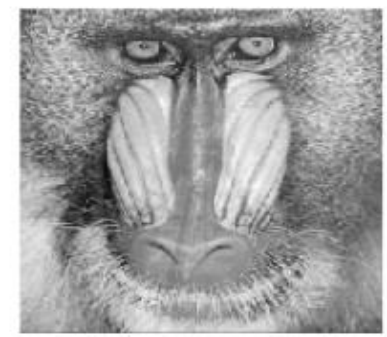

PDF distribution of y2\&y3

PDF distribution of $\mathrm{y} 1 \& \mathrm{y} 3$

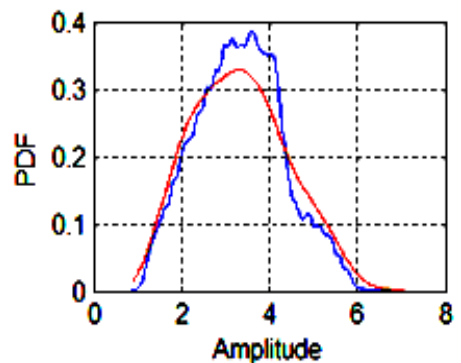

(d)

Fig.7 (a) mixed of 3 images, (b) recovered 3 images using nonlinear ICA method, (c) recovered 3 images using the proposed Entropy method, (d) $p d f$ distribution of the 3 recovered images by the proposed entropy method. 


\section{REFERENCES}

[1] J. A. Cadzow, "Blind deconvolution via cumulant extrema," pp. 24-42, Signal processing Mag., vol. 13, no. 3, May 1996.

[2] D. C. B. Chan, S. J. Godsill and P. J. W. Rayner, "Multi-channel blind signal separation by decorrelation," in Proc. Of IEEE Signal Processing Society 1995 Workshop on Applications of Signal Processing to Audio and Acoustics, Mohonk Mountain House, New York, pp. 15-18, Oct. 1995.

[3] D. C. B. Chan, S. J. Godsill and P. J. W. Rayner, "Multi-channel signal separation," on Proc. of ICASSP'96, vol. II, pp. 649-652, Atlanta, U. S. A, May 1996.

[4] M. F. Fahmy, G. M. El-Raheem and A. A. Abdel-Sallam, "Signal separation using second and high-order statistics," Int. Journal of Circuit Theory and Appl., Vol 28, no.3, pp. 225-243, May-June 2000.

[5] M. F. Fahmy , G. A. El-Raheem "A fast second order signal separation algorithmwith on-line capabilities," International Journal of Circuit Theory and Applications, Vol. 30, pp. 425-439, 2002.

[6] A. Cichocki and S. Amari, "Adaptive Blind Signal and Image Processing," John Wiley and Sons, LTD, 2002

[7] A. Hypavarinen, J. Karahunen and E. Joha," Independent Component Analysis," John Wiley and Sons, LTD, 2001

[8] A. Taleb, C. Jutten, "Batch algorithm for Source seperation in postnonlinear mixtures," Proc. ICA, Ausois. France, pp. 155-160, Jan. 1999.

[9] A. Taleb, C. Jutten, " Source seperation in postnonlinear mixtures," IEEE Transaction on Signal Processing, vol. 47, no. 10, pp. 2807-2820, October 1999,

[10] M. F. Fahmy, G. Abdel Raheem, U. S. Mohamed, Omar F. Fahmy and G. F. Fahmy, "Denoising and image compression using Bspline wavelets," Proc. of ISSPIT08, Sarayavio, Bosnia, Dec. 2008.

[11] A. Cichocki and S. Amari. Adaptive Blind Signal And Image Processing. John Wiley, New York, 2003.

[12] M. F. Fahmy, G. M. Fahmy and O. M. Fahmy, "Bspline wavelets in signal denoising and image compression," Journal of Signal, Image and Video Processing, Dec. 2009. 
الفصل المبهم لإثارات المخلوطة خيطا باستخدام الاستنتاج الدقيق لالة كثافة الاحتمالات

في هذا المقال نم تقديم طريقة جديدة للفصل المبهم لإثـارات المخلوطة خيطا. الفكرة الأساسية تعتمد علي الاستتناج الدقيق لدالة كثافة الاحتمالات (pdf) للإثارات بالإضافة إلي مسنوي الفوضي في الإنشارة (entropy) البيانات نظرا لميزة موجات ال Bspline الممتازة في تركيز الطاقة. وقد وجد أن هذه الفرضية دقيقه وقويه لتقدير دالة الكثافة الاحتمالية (pdf) التي تستخدم في تصميمات فصل الإنثارات المبهمة (Binear) BSS

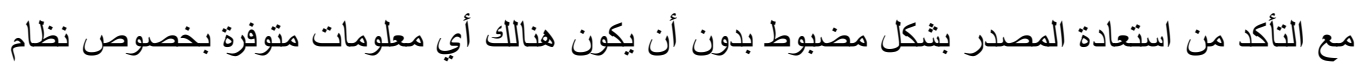

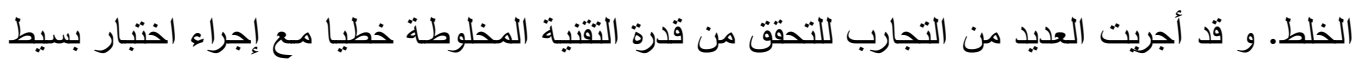

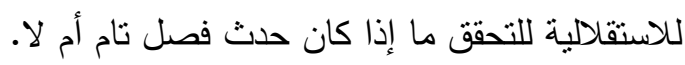

